This item was submitted to Loughborough's Research Repository by the author.

Items in Figshare are protected by copyright, with all rights reserved, unless otherwise indicated.

\title{
The control of twisting somersaults
}

PLEASE CITE THE PUBLISHED VERSION

http://dx.doi.org/10.1016/j.jbiomech.2014.02.006

PUBLISHER

(c) Elsevier

VERSION

AM (Accepted Manuscript)

LICENCE

CC BY-NC-ND 4.0

REPOSITORY RECORD

Yeadon, Fred, and Michael Hiley. 2014. "The Control of Twisting Somersaults". Loughborough University. https://hdl.handle.net/2134/14602. 


\title{
THE CONTROL OF TWISTING SOMERSAULTS
}

\author{
Maurice R. Yeadon and Michael J. Hiley \\ School of Sport, Exercise and Health Sciences, Loughborough University, LE11 3TU, UK
}

\begin{abstract}
In the takeoff and early flight phase of a twisting somersault, joint coordination is based on feedforward control whereas in the late stages of the flight phase configuration adjustments are made using feedback control to ensure accurate completion of the movement and appropriate landing orientation. The aim of this study was to use a computer simulation model of aerial movement to investigate the extent to which arm and hip movements can control twist and somersault rotation in the flight phase of a twisting somersault. Two mechanisms were considered for the control of twist in simulated target trampoline movements with flight times of $1.4 \mathrm{~s}$. In the first case a single symmetrical arm adduction correction was made using delayed feedback control based on the difference between the twist rate in a perturbed simulation and the twist rate in a target movement comprising a forward somersault with $1 \frac{1}{2}$ twists. Final corrections were made using symmetrical arm abduction and hip flexion to adjust the twist and somersault angles. In the second case continual asymmetrical arm adduction / abduction adjustments were used to remove the tilt from a perturbed full twisting backward somersault using delayed feedback control based on twist angle and angular velocity. The first method was able to cope with perturbations to a forward somersault with $1 \frac{1}{2}$ twists providing the feedback time delay was less than 200 ms. The second method was able to correct a perturbed full twisting backward somersault providing the feedback time delay was less than $125 \mathrm{~ms}$.
\end{abstract}

Keywords: computer simulation, aerial movement, technique, feedback control

\section{Introduction}

Targeted movements in sport may be coordinated using feed-forward control as in the throwing of a dart in which the action is completely preplanned. Alternatively the sports participant may have to react to changing circumstances as in a tennis rally in which feedback control is used to coordinate movement. In acrobatic movements with a flight phase, feed-forward control is used for coordination in the takeoff and early part of the flight phase. In the latter stages of the flight phase, feedback control is used to adjust body configuration in order to obtain the intended appropriate target landing orientation.

In acrobatic sports such as gymnastics and trampolining, twist may be introduced into a somersault during the contact phase (Yeadon, 1993a, 1993b). Alternatively twist may be initiated during the aerial phase by means of asymmetrical arm or hip movements (Yeadon, 1993c, 1993d). Such mechanisms for achieving a targeted movement are typically learned by repeated attempts (Schmidt, 1975). These learned joint movements are not identical from performance to performance since there is always variability in the execution of coordinated movements. This coordination variability arises from planning errors, execution errors and noise in the motor-sensory system (van Beers et al., 2004; Cohen and Sternad, 2009; Bartlett et al., 2007). As a consequence the somersault and twist resulting from the configuration changes also has variability and some form of feedback control is needed to reduce this outcome variability (Hiley et al., 2013).

Estimates of joint angle variability obtained from repeated giant circles on high bar by an elite gymnast range from $1^{\circ}$ to $3^{\circ}$ (Hiley et al., 2013). Mean angular velocity variability over the last half circle on high bar prior to a Tkatchev release by an elite gymnast was $1.3 \%$ (Hiley and Yeadon, 2012). It might be expected therefore that release velocity and angular momentum about the mass centre would have similar variability and that rotation potential (the product of angular momentum and flight time) would have variability of around $2 \%$. For a twisting somersault such variability in the initial conditions of flight 
may be expected to lead to similar variability in somersault but possibly greater variation in twist since joint movements will have a different effect when made at different twist values. It is to be expected that elite gymnasts will have lower variability in initial conditions and joint angle time histories and that this will lead to less variability in movement outcomes.

In the case of twisting somersaults the twist rate and somersault rate have the potential to be controlled using symmetrical changes in arm abduction and hip flexion. For aerial twists that arise from tilt produced by asymmetrical movements in the flight phase, the twist may be stopped prior to landing by removing the tilt at an integral number of half twists, again using asymmetrical movements of the arms and hips (Yeadon, 1993c). This provides another potential means for ensuring that the targeted final twist angle is achieved: by making adjustments to the tilt angle. The task of closely matching the intended target values of somersault, tilt and twist angles simultaneously at the time of landing is a complex one since configuration changes that affect one of these three angles also have some effect on the remaining two angles. The problem is aggravated by the inherent feedback system delay which can be up to 100 to $200 \mathrm{~ms}$ for long loop / triggered and voluntary responses (Latash, 1998). Thus any correction has to be based upon the state of the mechanical system at a previous time. As a consequence there will be limits to the level of control that can be exercised on the somersault and twist rotations.

When the landing area is viewed late in the flight phase there will be limited time in which to flex or extend the hips to adjust the somersault rate and hence orientation prior to landing. As a consequence it is likely that a single adjustment will be made. In contrast the build-up of twist in an unstable nontwisting straight double somersault may be controlled by asymmetrical arm movements using continual feedback throughout the flight phase (Yeadon and Mikulcik, 1996).

In this study the extent to which arm and hip movements can control twist and somersault during the aerial phase of a twisting somersault will be investigated for two hypothetical target movements using discrete and continuous automatic control schemes implemented within a computer simulation model of aerial movement (Yeadon et al., 1990).

\section{Method}

Two hypothetical simulated target movements were used to evaluate methods of controlling twist and somersault in aerial movements. Various perturbations were introduced into the original movements and in-flight corrections were made with the aim of achieving the target values of somersault, tilt and twist.

An 11-segment computer simulation model of aerial movement was used with the segmental inertia parameters of an elite trampolinist obtained from anthropometric measurements (Yeadon, 1990) to generate target simulations. The model had previously been evaluated against recorded performances in gymnastics: floor (Yeadon and Kerwin, 1999), high bar (Yeadon, 1997), rings (Yeadon, 1994), trampolining (Yeadon et al., 1990), diving (Yeadon, 1993e) and the aerials event in freestyle skiing (Yeadon, 1989).

\section{Discrete control}

The first target movement had duration $1.4 \mathrm{~s}$ and comprised a forward somersault with $1 \frac{1}{2}$ twists (as used in trampolining) produced by asymmetrical movement of the hips in the aerial phase (Figure 1). The body moved from a forward hips flexed position with arms abducted through side flexion over the right hip before extending to a straight body configuration, resulting in a twist to the left (Yeadon, 1993c). The minimum angle between the upper trunk and the thighs was $128^{\circ}$. The arm abduction angle during the middle phase in which the body was held straight was $9^{\circ}$. The tilt was removed using asymmetrical hip movement in which the body moved from a side flexed position over the left hip into a forward hips flexed position. As a consequence the twist stopped. Angle changes were made using a quintic function with zero first and second derivatives (Hiley and Yeadon, 2003).

Four perturbations were introduced into the twisting somersault using the variation levels described in the Introduction. In the first perturbation the maximum hip flexion was reduced by $2^{\circ}$, resulting in a decrease in the maximum tilt angle from $12.5^{\circ}$ to $11.5^{\circ}$, a decrease in the final twist angle from $540^{\circ}$ to 


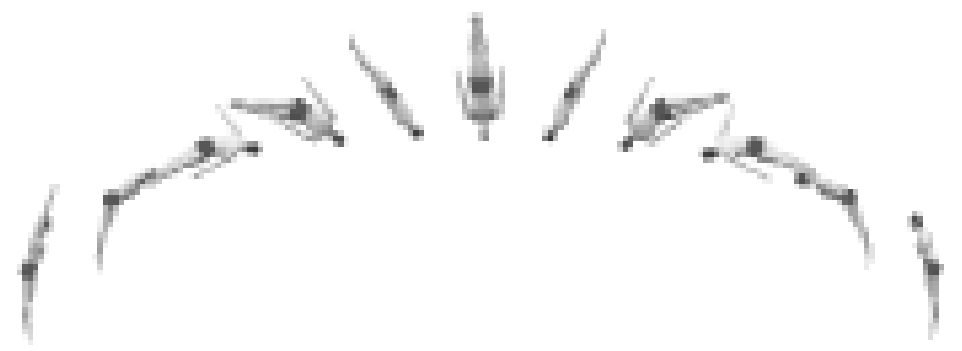

Figure 1: Forward $1 \frac{1}{2}$ twisting somersault target simulation with a flight time of $1.4 \mathrm{~s}$

$501^{\circ}$ and a decrease in the final somersault angle of $14^{\circ}$. In the second perturbation hip flexion was increased by $2^{\circ}$, resulting in an increase in the maximum tilt angle from $12.5^{\circ}$ to $13.4^{\circ}$, an increase in the final twist angle from $540^{\circ}$ to $618^{\circ}$ and an increase in the final somersault angle of $6^{\circ}$. In the third perturbation the (somersault) angular momentum was decreased by $2 \%$, resulting in reductions of $0.3^{\circ}, 23^{\circ}$ and $13^{\circ}$ in tilt, twist and somersault. In the fourth perturbation the somersault momentum was increased by $2 \%$, resulting in increases of $0.2^{\circ}, 31^{\circ}$ and $10^{\circ}$ in tilt, twist and somersault.

The twist rate $\dot{\psi}$ about the longitudinal axis of an axially symmetric body is given by $\dot{\psi}=[h / C-$ $h / A] \sin \theta$ where $h$ is the total angular momentum about the mass centre, $A$ is the transverse moment of inertia, $C$ is the longitudinal moment of inertia and $\theta$ is the tilt angle (Yeadon, 1993a). Thus adducting the arms during the central phase of the movement when the body is straight will produce a twist rate proportional to $[1 / C-1 / A]$. A decrease in arm adduction of $1^{\circ}$ from the $9^{\circ}$ in the target movement will correspond to an increase of $2 \%$ in the twist rate.

In order to correct for a perturbation there are the added complications of a shortfall of twist prior to the middle phase as well as the delay in feeding back twist rate information. The scheme for correcting for the twist error arising from a perturbation comprised changing the arm adduction angle in proportion to the percentage difference between the perceived twist rate in the perturbed simulation compared with that of the target movement at the time of the start of the middle phase with arms adducted at $9^{\circ}$ from the body. The adduction arm angles were each increased by $\delta$ where:

$\delta=p\left(\dot{\psi} / \dot{\psi}_{T}\right)$

with $p$ the constant of proportionality, $\dot{\psi}$ the twist rate at the start of the middle phase with arm abduction equal to $9^{\circ}$, and $\dot{\psi}_{T}$ the corresponding twist rate in the target movement. A quintic function with zero first and second derivatives was used to make the change in arm angle (Hiley and Yeadon, 2003). A time delay of $200 \mathrm{~ms}$ was used and so the arm angle did not start to change until $200 \mathrm{~ms}$ after the start of the middle phase. The duration of this (small) arm movement was set at $100 \mathrm{~ms}$. The last $100 \mathrm{~ms}$ of the middle phase was used to reset the arm adduction angle to $9^{\circ}$.

Constants of proportionality for the correction each of the four perturbations were determined (empirically) in order that the final twist angle matched the target value of $540^{\circ}$. Since a gymnast cannot tailor such a constant to an individual perturbation an average value was taken and corrective simulations were run again for the four perturbations. While the effects of the perturbations were reduced, the final twist angles no longer matched the target value. Additionally there were errors in the final somersault angle that would have made landing on trampoline less than ideal.

In the last part of the flight phase of a twisting somersault a gymnast is able to view the landing area and make judgements on the amounts of somersault and twist required in the remaining time as well as somersault and twist rates. Although there will be a feedback time delay a forward estimate of twist angle can be made using previous values of twist angle and twist rate. Rather than doing this it was assumed that final adjustment could be made on the basis of the twist angle 200 ms before landing without a time delay. Arm adduction was changed in proportion to the percentage change in the amount of remaining twist compared with the target movement $200 \mathrm{~ms}$ before landing. The constant of proportionality for the final twist correction was determined empirically by running numerous simulations for the four perturbations. The duration of arm movement was set at $200 \mathrm{~ms}$. A similar procedure was used to adjust the somersault angle by changing the amount of hip flexion over the last $200 \mathrm{~ms}$ based on the somersault angle. 


\section{Continuous control}

The second target movement comprised a backward somersault with a full twist produced during the $1.4 \mathrm{~s}$ aerial phase using asymmetrical arm movements (Figure 2). From an initial position with each arm abducted $90^{\circ}$ from the body, the left arm was adducted through $90^{\circ}$ to the side of the body while the right arm was abducted $90^{\circ}$ to an overhead position. The arms remained in this asymmetrical position until $\hat{\mathrm{A}}_{4}^{3}$ revolutions of twist were completed after which point the arms were spread to be symmetrical once again, removing the tilt and stopping the twist. Angle changes were made using a quintic function with zero first and second derivatives (Hiley and Yeadon, 2003).

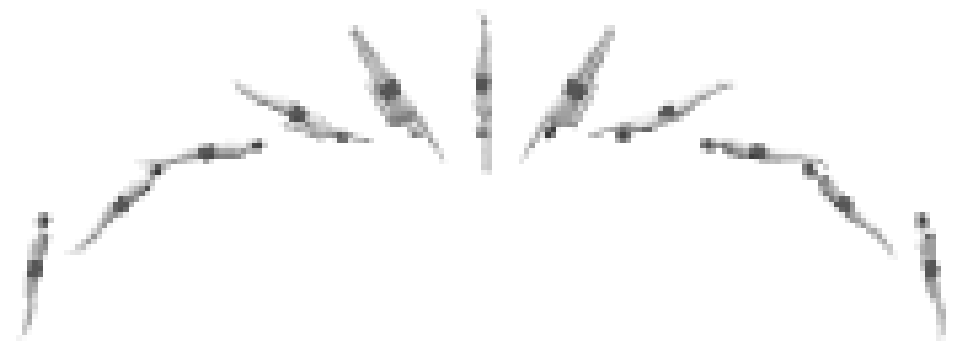

Figure 2: Backward full twisting somersault target simulation with a flight time of $1.4 \mathrm{~s}$

Four perturbations were introduced into the twisting somersault. In the first perturbation the arm adduction/abduction was reduced by $10^{\circ}$, resulting in an increase in the tilt angle at the half twist position from $8.9^{\circ}$ to $9.5^{\circ}$, a decrease in the final twist angle from $360^{\circ}$ to $336^{\circ}$ and a decrease in the final somersault angle of $4^{\circ}$. In the second perturbation arm movement was made $0.03 \mathrm{~s}$ earlier, resulting in no change in the tilt angle, an increase in the final twist angle from $360^{\circ}$ to $388^{\circ}$ and an increase in the final somersault angle of $0.5^{\circ}$. In the third perturbation the (somersault) angular momentum was decreased by $2 \%$, resulting in reductions of $0.0^{\circ}, 13^{\circ}$ and $8^{\circ}$ in tilt, twist and somersault. In the fourth perturbation the (somersault) angular momentum was increased by $2 \%$, resulting in increases of $0.0^{\circ}$, $24^{\circ}$ and $11^{\circ}$ in tilt, twist and somersault.

In order to correct for each perturbation the scheme described for non-twisting straight somersaults in Yeadon and Mikulcik (1996) was implemented in which the corrective arm adduction / abduction change over a simulation time step was a proportional plus derivative function of the twist angle at an earlier time. For non-twisting straight somersaults the twist angle was controlled continuously to remain close to zero whereas in the current movement the aim was to control the twist to reach $360^{\circ}$ and remain close to this value. The arm abduction angles were changed by $\delta$ and-delta over a simulation time step according to:

$\delta=p \psi+d \dot{\psi}$

where $p$ and $d$ are constants, $\psi$ is the twist angle and $\dot{\psi}$ is the twist angular velocity. The corrections were based on twist angle and angular velocity data starting from two thirds way through the simulation when the twist angle in the target simulation would have been $90 \%$ complete and the arm adduction/abduction angles would have changed by $8^{\circ}$ from their midflight values. Implementation started later than this by the feedback delay time. For $100 \mathrm{~ms}$ delay the twist angle in the target simulation would have been $98 \%$ complete and the arm adduction/abduction angles would have changed by $51^{\circ}$ from their midflight values. In order to assess whether the control was stable the simulation was run for an additional somersault. If the arm oscillation amplitude decreased the control was termed stable; if it increased it was termed unstable. The feedback time delay was set initially to $100 \mathrm{~ms}$ and was then increased up to the point at which control was no longer stable. Constants of proportionality for each of the four perturbations were determined (empirically) in order that stable control of the twist angle was achieved for a feedback delay time of $100 \mathrm{~ms}$. A single pair of proportional plus derivative constants were then found for which stable control was achieved for each of the four perturbations. These values were adjusted as the time delay was increased incrementally from $100 \mathrm{~ms}$ until control was no longer stable. 


\section{Results}

The four perturbations to the $11 / 2$ forward twisting somersault (the first target movement) resulted in twist angle errors ranging from $-39^{\circ}$ to $+78^{\circ}$ away from the target twist angle of $540^{\circ}$. When the correction scheme based on the mid-phase twisting velocity was applied, the error range decreased to $-5^{\circ}$ to $+9^{\circ}$. When the additional correction based on the final phase twist angle was made, the twist error range decreased further to $-2^{\circ}$ to $+4^{\circ}$ (Table 1). The somersault angle errors arising from the four perturbations ranged from $-14^{\circ}$ to $+13^{\circ}$. When the twisting velocity correction was applied, the error range decreased to $-6^{\circ}$ to $+6^{\circ}$ and when the twist angle and somersault angle corrections were made, the somersault error range decreased further to $-3^{\circ}$ to $-1^{\circ}$ (Table 2 ).

Table 1: Twist angle errors [in ${ }^{\circ}$ ] in perturbed $1 \frac{1}{2}$ twisting forward somersault arising from changes in hip flexion and angular momentum

\begin{tabular}{|l|c|c|c|c|}
\hline perturbation & $-2^{\circ}$ hip & $+2^{\circ}$ hip & $-2 \%$ AM & $+2 \%$ AM \\
\hline uncorrected & -39 & 78 & -23 & 31 \\
\hline correction 1 & -5 & 9 & 4 & -3 \\
\hline correction 2 & -2 & 4 & 0 & 1 \\
\hline
\end{tabular}

Correction 1 is based on twist velocity in the mid-phase

Correction 2 is based on twist angle in the final phase

Table 2: Somersault angle errors [in ${ }^{\circ}$ ] in perturbed $1 \frac{1}{2}$ twisting forward somersault arising from changes in hip flexion and angular momentum

\begin{tabular}{|l|c|c|c|c|}
\hline perturbation & $-2^{\circ}$ hip & $+2^{\circ}$ hip & $-2 \% \mathrm{AM}$ & $+2 \% \mathrm{AM}$ \\
\hline uncorrected & -14 & 6 & -13 & 10 \\
\hline correction 1 & -2 & 2 & -6 & 6 \\
\hline correction 2 & -1 & -3 & -2 & -2 \\
\hline
\end{tabular}

Correction 1 is based on twist velocity in the mid-phase

Correction 2 is based on twist angle in the final phase

In the first perturbation the twist and somersault deviations were $-39^{\circ}$ and $-14^{\circ}$ respectively. These decreased to $-2^{\circ}$ and $-1^{\circ}$ when the corrections were made (Figures 3 and 4 ). To effect the twisting velocity correction, the arm abduction angle in mid-phase was reduced from $+9^{\circ}$ to $-4^{\circ}$ (Figure 5). To effect the final twist and somersault corrections, the arm and hip angles were adjusted by $19^{\circ}$ and $3^{\circ}$ respectively (Figures 3 and 5).

In the second perturbation the twist deviation was $+78^{\circ}$ which was corrected by increasing the arm abduction angle in the mid-phase from $9^{\circ}$ to $21^{\circ}$ (Figure 6 ). To effect the final twist and somersault corrections (Tables 1 and 2), the arm and hip angles were adjusted by $34^{\circ}$ and $3^{\circ}$ (Figure 6 ).

The four perturbations to the full twisting backward somersault (the second target movement) resulted in twist angle errors ranging from $-46^{\circ}$ to $+28^{\circ}$ away from the target twist angle of $360^{\circ}$. When the proportional plus derivative control scheme was applied for a feedback time delay of $100 \mathrm{~ms}$, the twist error range decreased to $0^{\circ}$ to $2^{\circ}$ (Table 3 ). When the feedback delay time was increased a limit of $125 \mathrm{~ms}$ was reached beyond which twist control was not stable. For this time delay the twist error range was $0^{\circ}$ to $4^{\circ}$ (Table 3 ). Since no attempt was made to correct somersault errors, these remained largely unchanged by the twist corrections (Table 4).

In the first perturbation the twist deviation was $-46^{\circ}$ which decreased to $0^{\circ}$ when the $100 \mathrm{~ms}$ delay correction was made (Figures 7 and 8). The arm asymmetry at the full twist position was $64^{\circ} / 116^{\circ}$ (Figure 9) while in the subsequent (virtual) somersault this decreased to $89^{\circ} / 91^{\circ}$, indicating that control was stable.

In the second perturbation the twist deviation was $+28^{\circ}$ which decreased to $2^{\circ}$ when the $100 \mathrm{~ms}$ delay correction was made (Table 3 ). The arm asymmetry at the full twist position was $66^{\circ} / 114^{\circ}$ (Figure 10) while in the subsequent (virtual) somersault this decreased to $82^{\circ} / 98^{\circ}$. 


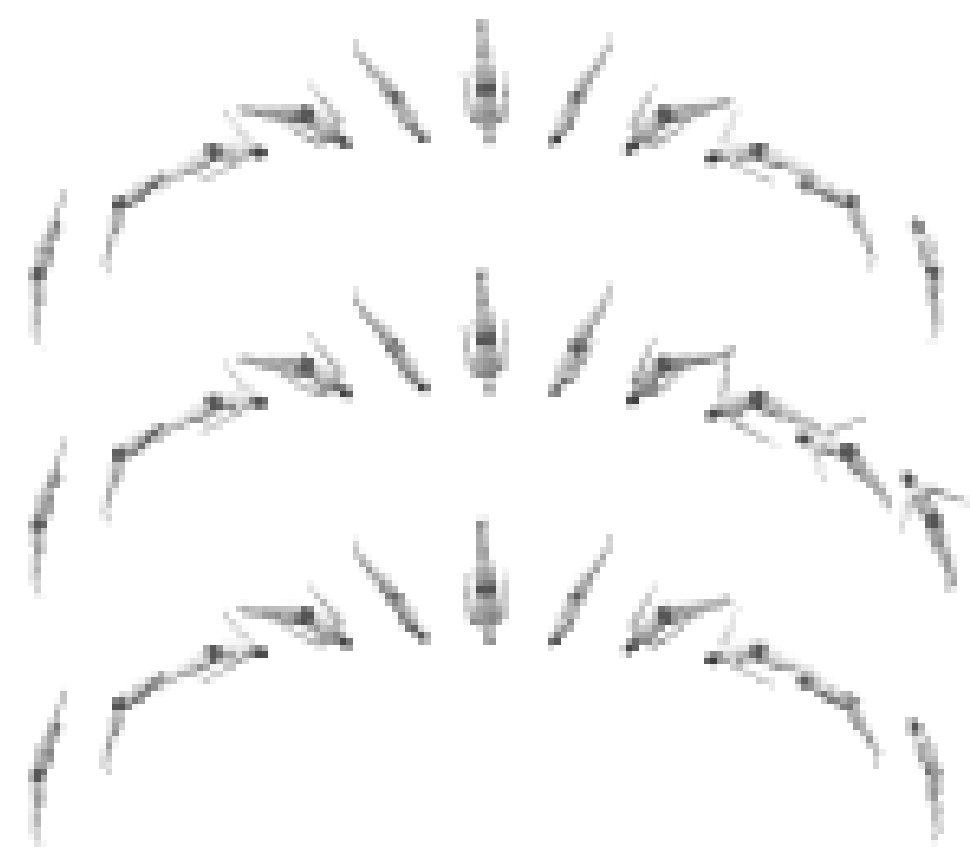

Figure 3: Graphics of target $1 \frac{1}{2}$ twisting somersault (upper sequence), perturbation arising from less hip flexion (middle sequence), corrected simulation (lower sequence).

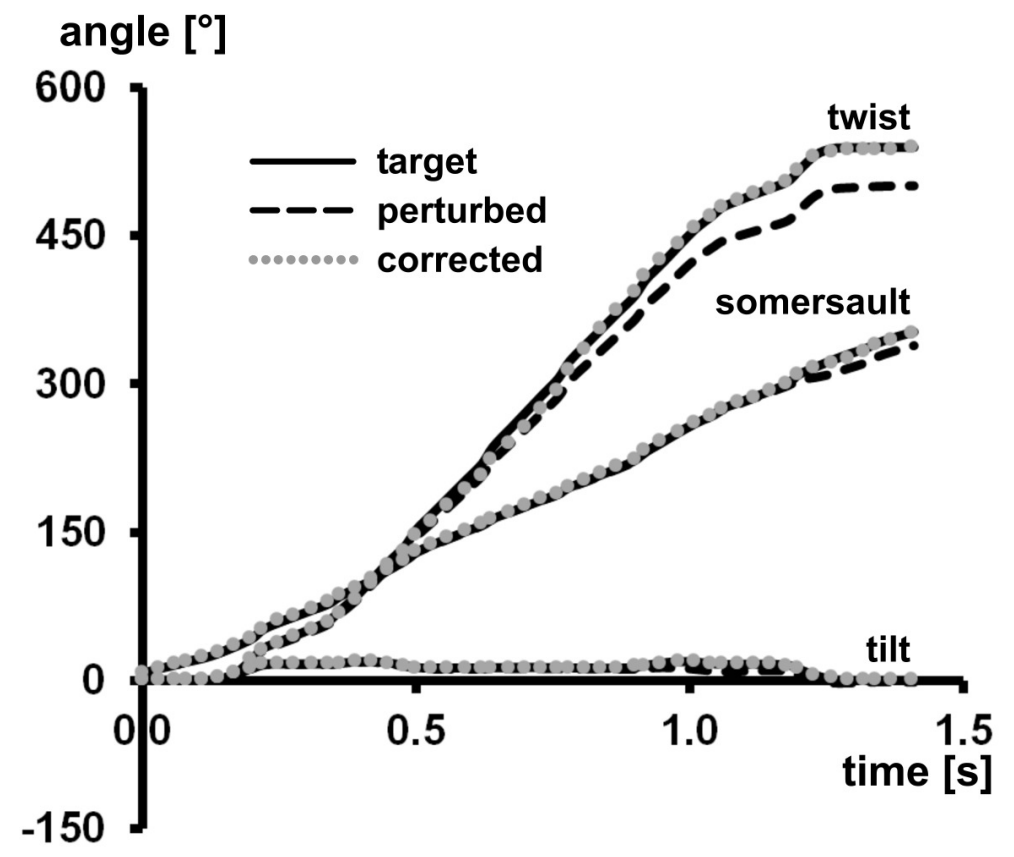

Figure 4: Comparisons of the time histories of somersault, tilt and twist angles for the $1 \frac{1}{2}$ twisting somersault: target simulation (solid lines), perturbed simulation (dashed lines) and corrected simulation (dotted lines). 


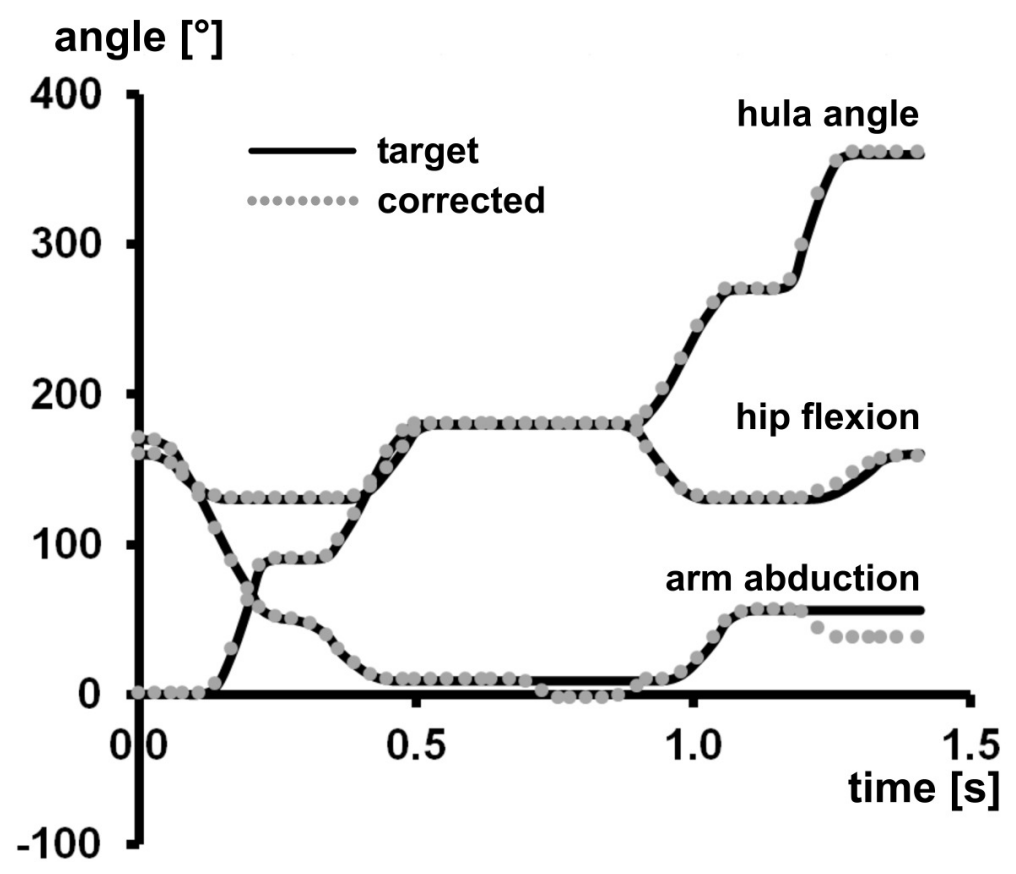

Figure 5: Comparisons of the time histories of arm and hip angles for the $1 \frac{1}{2}$ twisting somersault: target simulation (solid lines), corrected simulation (dotted lines).

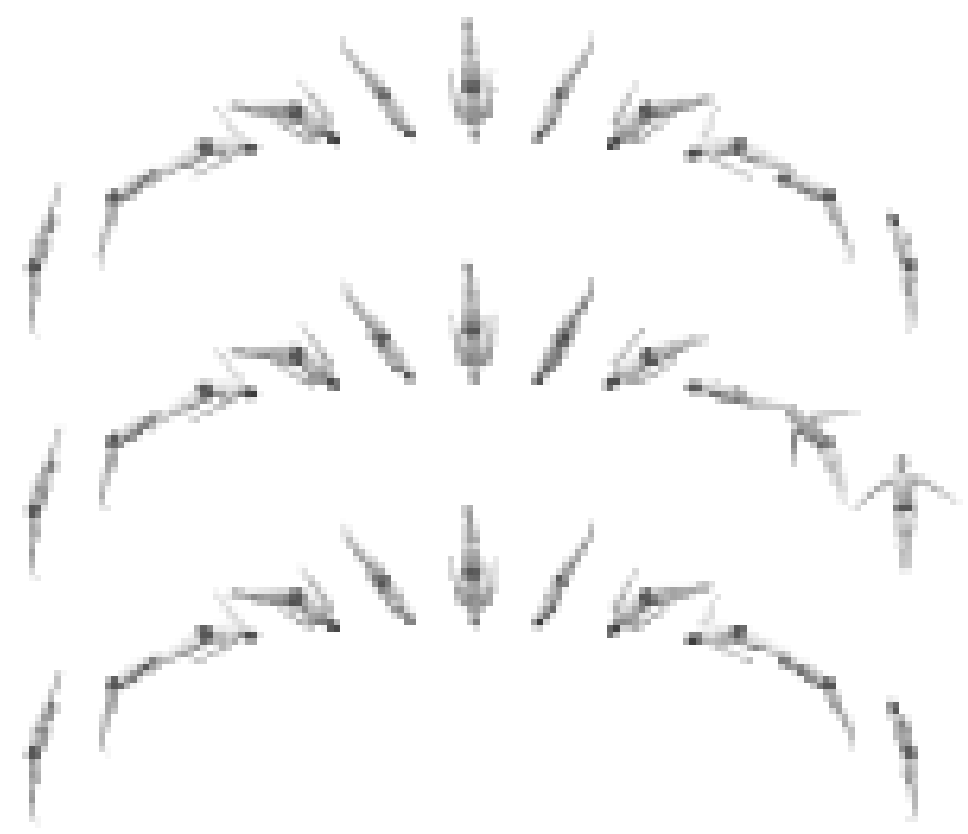

Figure 6: Graphics of target $1 \frac{1}{2}$ twisting somersault (upper sequence), perturbation arising from more hip flexion (middle sequence), corrected simulation (lower sequence). 


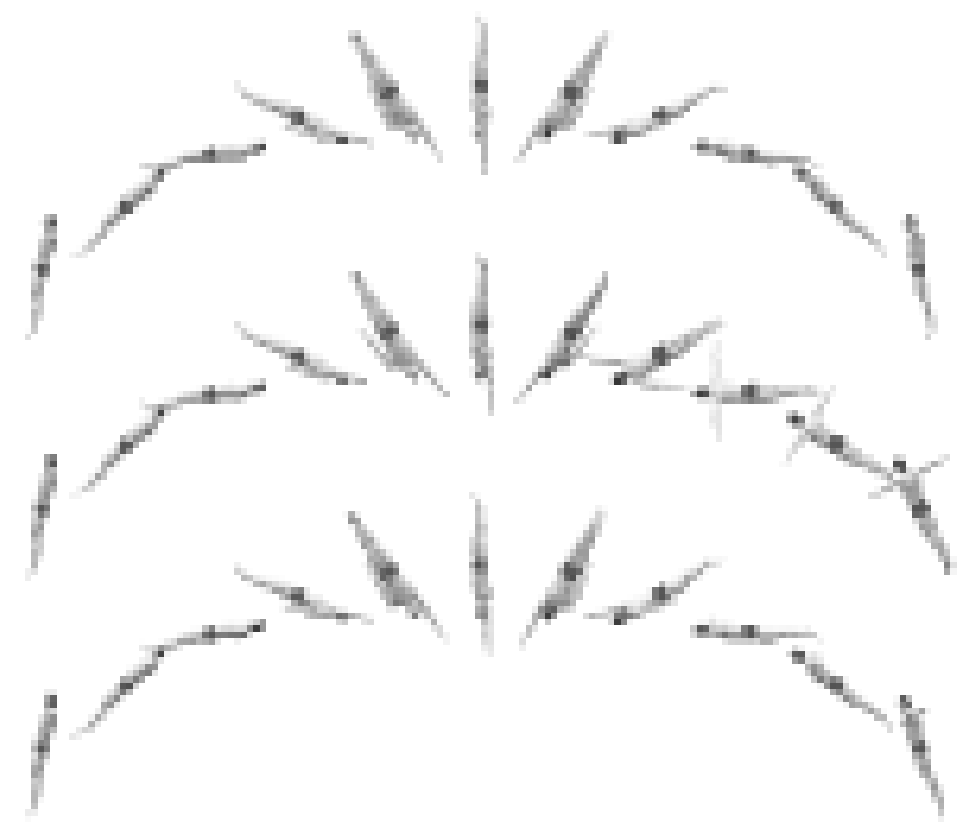

Figure 7: Graphics of target full twisting somersault (upper sequence), perturbation arising from less arm abduction (middle sequence), corrected simulation (lower sequence).

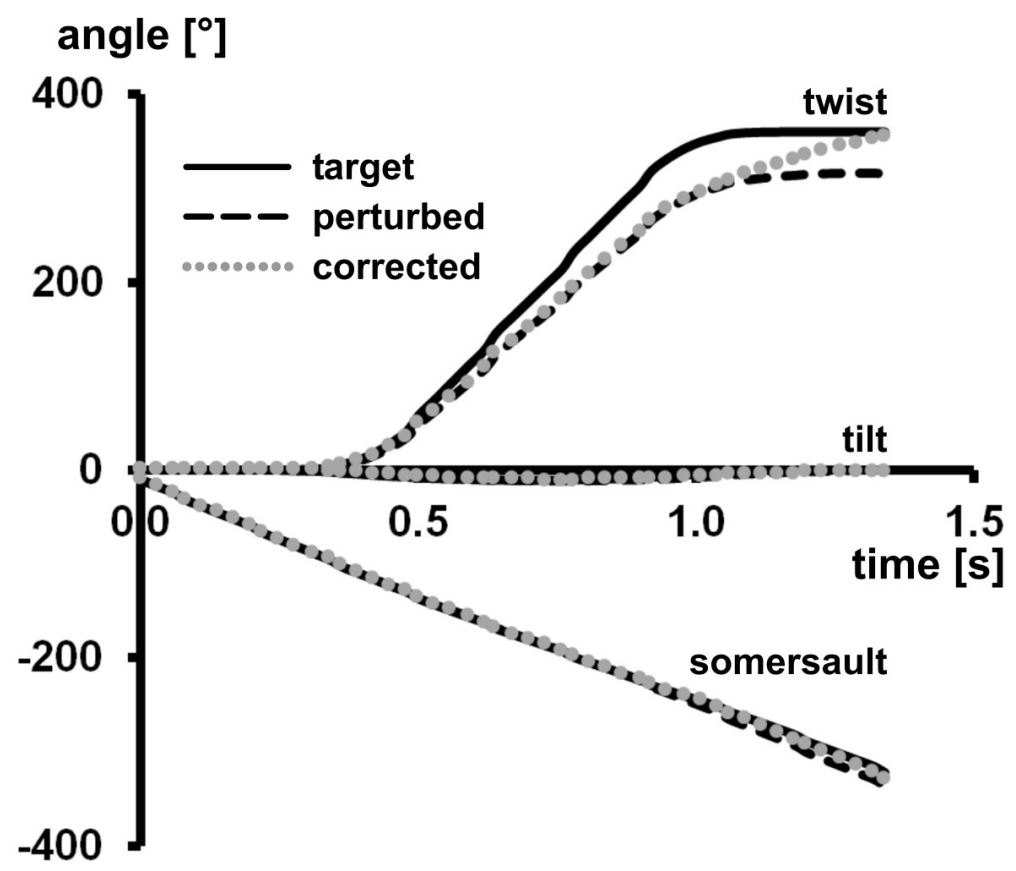

Figure 8: Comparisons of the time histories of somersault, tilt and twist angles for the full twisting somersault: target simulation (solid lines), perturbed simulation (dashed lines) and corrected simulation (dotted lines). 


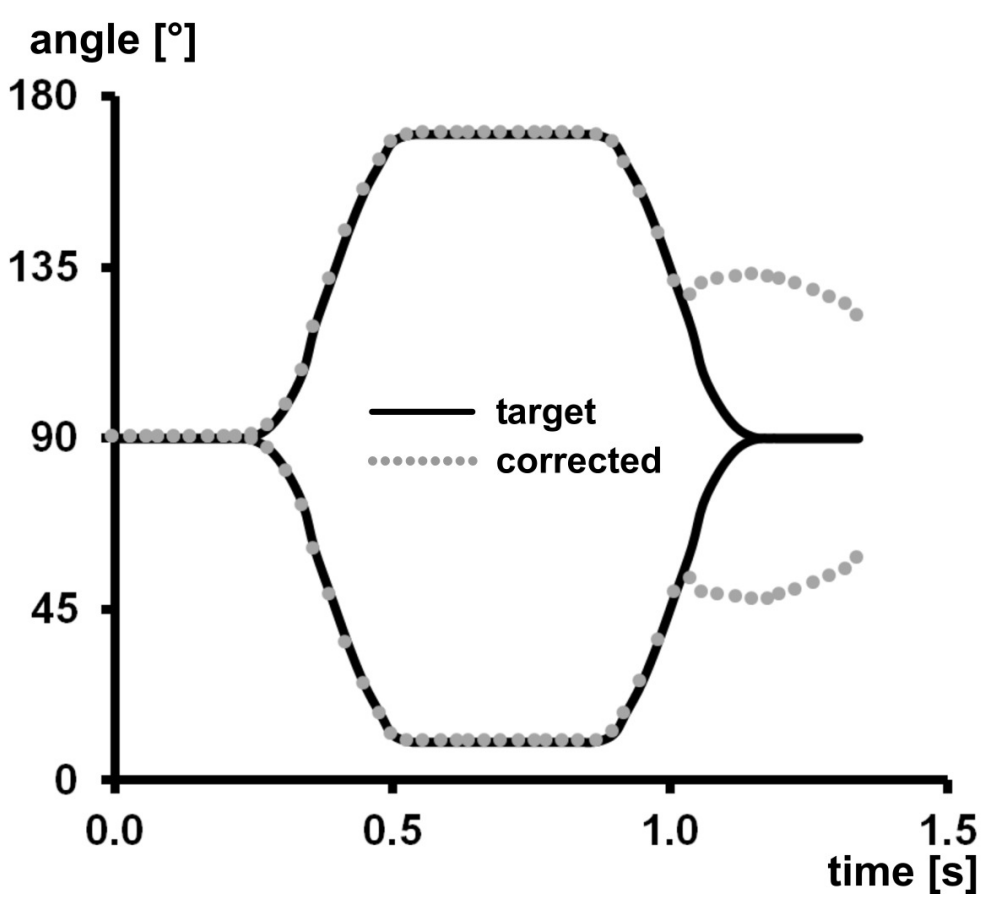

Figure 9: Joint angles for target and corrected full twisting somersault.

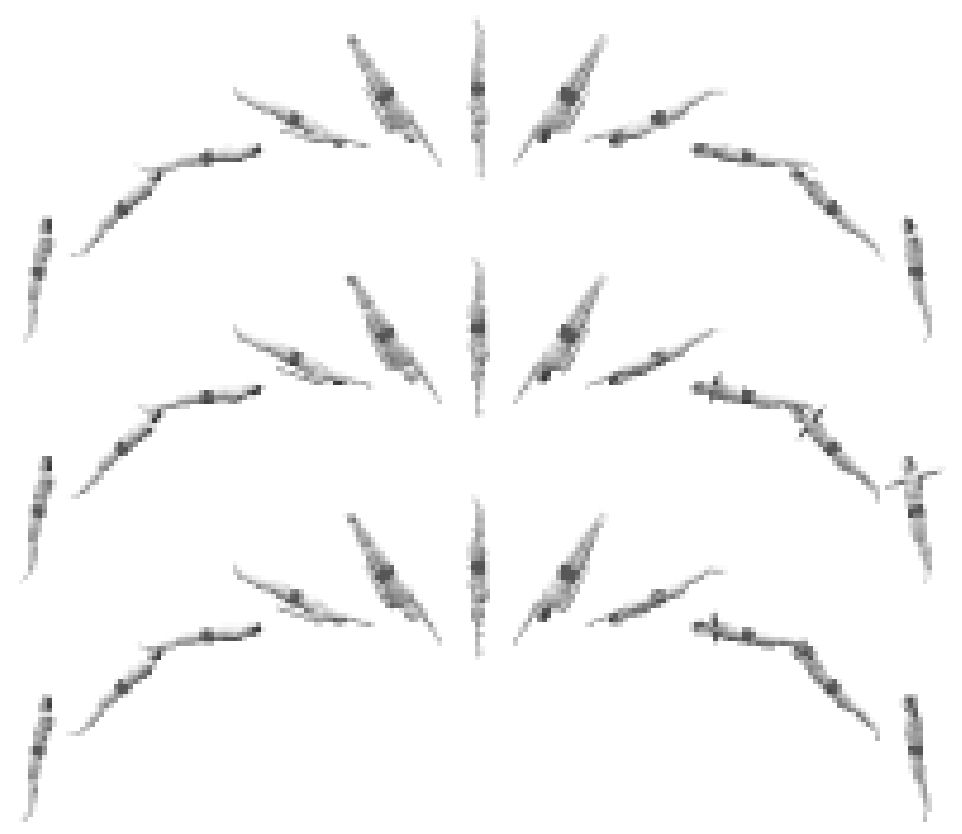

Figure 10: Graphics of target full twisting somersault (upper sequence), perturbation arising from earlier arm abduction (middle sequence), corrected simulation (lower sequence). 
Table 3: Twist angle errors $\left[\right.$ in $^{\circ}$ ] in perturbed full twisting backward somersault arising from changes in arm abduction and angular momentum

\begin{tabular}{|l|r|c|c|c|}
\hline perturbation & $-10^{\circ}$ & $-0.03 s$ arm & $-2 \%$ AM & $+2 \%$ AM \\
\hline uncorrected & -46 & 28 & -13 & 24 \\
\hline $100 \mathrm{~ms}$ correction & 0 & 2 & 2 & 2 \\
\hline $125 \mathrm{~ms}$ correction & 4 & 0 & 1 & 2 \\
\hline
\end{tabular}

Table 4: Somersault angle errors [in ${ }^{\circ}$ ] in perturbed full twisting backward somersault arising from changes in arm abduction and angular momentum

\begin{tabular}{|l|c|c|c|c|}
\hline perturbation & $-10^{\circ}$ & $-0.03 \mathrm{~s}$ & $-2 \%$ AM & $+2 \%$ AM \\
\hline uncorrected & -5 & 1 & -8 & 11 \\
\hline 100 ms correction & -8 & 2 & -7 & 12 \\
\hline 125 ms correction & -8 & 2 & -8 & 12 \\
\hline
\end{tabular}

\section{Discussion}

The forward $1 \frac{1}{2}$ twisting somersault was able to be controlled using arm abduction or adduction in the middle phase to adjust the twist rate with final corrections made on the basis of the twist and somersault angles. Since there is a limit to the amount of arm adduction that can be made in the middle phase, there will be a limit to the feedback delay time that can be accommodated. In the current example arm adduction reached $-4^{\circ}$, when correcting for a slow twist in the first perturbation, and so the method would be unable to cope with delays greater than the $200 \mathrm{~ms}$ used. When the perturbation is such that the twist rate is greater than needed, there is less of a problem since there is ample room for greater arm abduction.

This mid-phase strategy correction was able to reduce the twist perturbation by about a factor of 10 (Table 1). Achieving this level of correction is dependent on an accurate estimation of twisting velocity in comparison to the target velocity together with an appropriate strategy for the amount of correction to be made. Since there will be both planning and execution errors in the implementation of such a scheme (van Beers et al., 2004), it is to be expected that the errors remaining after the initial correction may be somewhat larger than those indicated in Table 1. As a consequence the need for final adjustment will be greater in practice than suggested here. The use of visual orientation information for this final adjustment is supported by the findings of Rézette and Amblard (1985).

The backward somersault with full twist was able to be controlled using asymmetrical arm adduction / abduction towards the end of the movement. The control scheme was based on making arm angle changes over a simulation time step as a proportional plus derivative function of the twist angle at an earlier time. This is equivalent to basing arm accelerations on twist angular velocity and twist angular acceleration estimates that may be provided by the inner ear balance mechanisms (Wendt, 1951). There was a limit of $125 \mathrm{~ms}$ to the feedback time delay for which stable control of the twist angle was possible. This corresponds to 0.09 (straight) somersaults. In the case of non-twisting straight somersaults a delay of 0.24 somersaults can be accommodated providing the arm asymmetry is less than $1^{\circ}$ (Yeadon and Mikulcik, 1996). For an arm asymmetry of $10^{\circ}$ and a delay of 0.12 somersaults the control was neutrally stable (the amplitude of arm movement remained constant). In the present case of controlling a twisting somersault, the arm asymmetry can be much larger than $10^{\circ}$ and so the delay of 0.09 somersaults for stable control is consistent with the earlier study of Yeadon and Mikulcik (1996). For straight double backward somersaults in a floor exercise in gymnastics, the flight time can be as low as $0.8 \mathrm{~s}$ (Hwang et al., 1990; Kerwin et al., 1998) and a delay of 0.24 somersaults would correspond to $100 \mathrm{~ms}$. This indicates that a time delay of $100 \mathrm{~ms}$ is feasible and so the limit of $125 \mathrm{~ms}$ for the correction of the theoretical full twisting somersault is able to be met in practice.

It is likely that this method of twist correction is learned during the practice of single straight somersaults with and without twist, and as a consequence when straight double somersaults without twist 
are attempted for the first time, the build-up of twist will be automatically resisted using this method. This description of how twist control is learned follows the stages of motor learning outlined by Fitts and Posner (1967). If this were not the case then the occurrence of an unexpected half twist when initially attempting a non-twisting straight double somersault might be expected to be a regular event. Gymnasts are typically unaware that they make such adjustments during non-twisting straight double somersaults or twisting somersaults. When a gymnast is operating at the autonomous stage (Fitts and Posner, 1967) it is unlikely that the movements will be performed at the conscious level. Indeed the associated time delays suggest that these actions are performed using long loop / triggered and voluntary responses (Latash, 1998). Coaches are also typically unaware of the need for correction and do not incorporate such knowledge into their coaching; for example, it is of benefit to view the landing area throughout a twisting double somersault.

When a gymnast attempts the same skill a number of times, no two attempts will be exactly the same due to the inherent variability in human movement (Bartlett et al., 2007). van Beers et al. (2004) described three sources of the variability in targeted tasks as being due to errors in the estimation of initial conditions, errors in planning the movement and execution errors. In the present study perturbations were introduced to target movements and so could be representative of all three sources of error. It has been demonstrated that through practice the cost of movement variability to the performance outcome can be reduced (Cohen and Sternad, 2009). However, even within the movements of highly trained elite gymnasts, movement variability will still exist (Cohen and Sternad, 2009; Hiley et al., 2013). One solution may be to adopt a technique that is able to cope with the level of variability within the movements, i.e. to operate within a solution space that is insensitive to the variability present (Cohen and Sternad, 2009; Hiley and Yeadon, 2013). However, in the present study such an approach is likely to be impossible but the movements are of sufficient duration to permit the detection of errors in the performed movement and take corrective measures.

The question of whether elite performance is characterised by low variability or high variability is often posed (Hiley et al., 2013; Wilson et al., 2008). The answer is that elite performance is characterised by both low variability and high variability. As can be seen from this study the outcome variability is low: i.e. the final somersault and twist values are close to the intended target values. The initial conditions variability is low: i.e. the takeoff velocity and angular momentum are close to the intended values. When corrections are made during flight these will vary from performance to performance and so there will be considerable variation in joint angle time histories in these phases. However in an individual performance such adjustments will be made with little variation from the intended adjustment. Thus elite gymnasts will typically make accurate movements at all times but because adjustments are needed in individual movements there will be variation from performance to performance.

\section{Conflict of Interest Statement}

There are no issues of conflict of interest arising from the personal or professional associations of the authors.

\section{References}

Bartlett, R., Wheat, J., Robins, M., 2007. Is movement variability important for sports biomechanists? Sports Biomechanics 6, 224-243.

Avan Beers, R.J., Haggard, P., Wolpert, D.M., 2004. The role of execution noise in movement variability. Journal of Neurophysiology 91, 1050-1063.

Cohen, R.G., Sternad, D., 2009. Variability in motor learning: relocating, channeling and reducing noise. Experimental Brain Research 193, 69-83.

Fitts, P.M., Posner, M.I., 1967. Human performance. Belmont: Brookes-Cole. 
Hiley, M.J., Yeadon, M.R., 2003. Optimum technique for generating angular momentum in accelerated backward giant circles prior to a dismount. Journal of Applied Biomechanics 19, 119-130.

Hiley, M.J., Yeadon, M.R., 2012. Achieving consistent performance in a complex whole body movement: The Tkatchev on high bar. Human Movement Science 31, 834-843.

Hiley, M.J., Yeadon, M.R., 2013. Investigating optimal technique in a noisy environment: application to the upstart on uneven bars. Human Movement Science 32, 181-191.

Hiley, M.J., Zuevsky, V., Yeadon, M.R., 2013. Is skilled technique characterised by high or low variability - An analysis of high bar giant circles? Human Movement Science 32, 171-180.

Hwang, I., Seo, G., Liu, Z.G., 1990. Take-off mechanics of the double backward somersault. International Journal of Sport Biomechanics 6, 177-186.

Kerwin, D.G., Webb, J., M.R. Yeadon., 1998. Production of angular momentum in double somersaults. In Proceedings of $\mathrm{XV}^{\text {th }}$ International Symposium on Biomechanics in Sports. University of Konstanz, Germany.

Latash, M.L., 1998. Neurophysiological basis of movement. Human Kinetics, Champaign, IL, pp. 98105.

Rézette, D, Amblard B., 1985. Orientation versus motion visual cues to control sensorimotor skills in some acrobatic leaps. Human Movement Science 4, 297-306.

Schmidt, R.A., 1975. A schema theory of discrete motor skill learning. Psychological Review 82, 225260.

Wendt, G.R., 1951. Vestibular functions. In: Stevens, S.S. (Ed.), Handbook of Experimental Psychology. Wiley, New York, pp. 1191-1223.

Wilson, C., Simpson, S.E., van Emmerik, R.A., Hamill, J., 2008. Coordination variability and skill development in expert triple jumpers. Sports Biomechanics 7, 2-9.

Yeadon, M.R., 1989. Twisting techniques used in freestyle aerial skiing. Journal of Sport Biomechanics, $5,275-281$.

Yeadon, M.R., 1990. The simulation of aerial movement - II: A mathematical inertia model of the human body. Journal of Biomechanics 23, 67-74.

Yeadon, M.R., 1993a. The biomechanics of twisting somersaults. Part I: Rigid body motions. Journal of Sports Sciences 11, 187-198.

Yeadon, M.R., 1993b. The biomechanics of twisting somersaults. Part II: Contact twist. Journal of Sports Sciences 11, 199-208.

Yeadon, M.R., 1993c. The biomechanics of twisting somersaults. Part III: Aerial twist. Journal of Sports Sciences 11, 209-218.

Yeadon, M.R., 1993d. The biomechanics of twisting somersaults. Part IV: Partitioning performance using the tilt angle. Journal of Sports Sciences 11, 219-225.

Yeadon, M.R., 1993e. Twisting techniques used by competitive divers. Journal of Sports Sciences 11, 4, 337-342. 
Yeadon, M.R., 1994. Twisting techniques used in dismounts from rings. Journal of Applied Biomechanics $10,2,178-188$.

Yeadon, M.R., 1997. Twisting double somersault high bar dismounts. Journal of Applied Biomechanics 13, 76-87.

Yeadon, M.R., Atha, J., Hales, F.D., 1990. The simulation of aerial movement - IV: A computer simulation model. Journal of Biomechanics, 23, 85-89.

Yeadon, M.R., Kerwin, D.G., 1999. Contributions of twisting techniques used in backward somersaults with one twist. Journal of Applied Biomechanics 15, 152-165.

Yeadon, M.R., Mikulcik, E.C., 1996. The control of non-twisting somersaults using configurational changes. Journal of Biomechanics 29, 1341-1348. 